\title{
Successful coronary lithotripsy after unsuccessful rotational atherectomy - use of complementary tools for treatment of undilatable calcified coronary artery stenosis
}

\author{
Piotr M. Wańczura ${ }^{1}$, Wojciech Wojakowski ${ }^{2}$ \\ ${ }^{1}$ Department of Cardiology, The Ministry of Internal Affairs and Administration, Hospital, Rzeszow, Poland \\ ${ }^{2}$ Department of Cardiology and Structural Heart Diseases, Medical University of Silesia, Katowice, Poland
}

Adv Interv Cardiol 2021; 17, 2 (64): 228-229

DOI: https://doi.org/10.5114/aic.2021.107507

Despite the progress in percutaneous treatment of coronary artery disease, massively calcified atherosclerotic lesions still constitute a challenge. Rotational atherectomy, capable of modifying the atherosclerotic plaque, known as debulking, has become the gold standard in the treatment of calcified lesions [1]. Recently, intravascular lithotripsy (IVL) became an alternative treatment option for undilatable calcified vessels. This technique uses a balloon-mounted ultrasound source which emits sonic pressure waves propagating through the fluid and delivered to the vessel wall. It results in fragmentation of superficial and deep calcium deposits allowing balloon expansion [2]. One limitation of this method is the relatively stiff, high-profile balloon, with lower crossability than regular semi- or non-compliant balloons routinely used during coronary angioplasty, especially in tight calcified lesions. Therefore effective IVL requires predilatation and may not be possible in uncrossable lesions. On the other hand, rotational atherectomy, even if performed with burs of increasing diameter, may be less effective in large-caliber vessels with calcifications located in the media. In such cases, an effective method combining initial debulking with rotational atherectomy followed by IVL may be an example of a comprehensive approach overcoming the limitations of both methods. IVL should be considered when rotational atherectomy, despite the use of a sufficiently large burr, is ineffective. The criteria for such a comprehensive approach to the massively calcified lesion in right coronary artery (RCA) were met by a 73-year-old patient with arterial hypertension, hyperlipidemia, persistent atrial fibrillation, and history of myocardial infarction treated with left anterior descending (LAD) percutaneous coronary intervention $(\mathrm{PCl})$ followed by elective recanalization of the circumflex chronic total occlusion (CTO). Due to persistent angina (CCS III class), the patient was qualified for $\mathrm{PCl}$ of the RCA. The predilatation with the NC balloon $(3.5 \times 15 \mathrm{~mm})$ was not successful due to the calcified undilatable lesion located in segment 2 of the RCA. We performed rotational atherectomy with the $1.5 \mathrm{~mm}$ and then $1.75 \mathrm{~mm}$ burrs (Figure $1 \mathrm{~A})$. Despite the high pressure (24 atm) inflation of the $3.5 \times 20 \mathrm{~mm}$ NC balloon, full expansion was not achieved (Figure $1 \mathrm{~B}$ ). There was dog-boning of the balloon and non-flow limiting dissection. The patient was clinically stable, and a second attempt with IVL was planned after 4 weeks. The procedure was performed using a $3.5 \times 12 \mathrm{~mm}$ IVL balloon (Shockwave Medical), which, after completing lithotripsy cycles, allowed for full expansion of the balloon at $6 \mathrm{~atm}$ (Figure $1 \mathrm{C}$ ). Finally, 2 drug-eluting stents (Promus $3.5 \times 24$ and Promus 3.5 $\times 20 \mathrm{~mm}$ ) were implanted with an optimal angiographic effect (Figure $1 \mathrm{D})$.

IVL is a valuable supplement to the available revascularization toolbox, consisting of rotational atherectomy, ultra high-pressure balloons, scoring, and cutting balloons. In the case of lesions that are too tight for the delivery of the lithotripsy balloon and, at the same time, not susceptible to expansion despite debulking with the available burr sizes, a hybrid approach based on a combination of both methods may be the only effective solution. Coronary aortic bypass surgery always remains an alternative, although lesions not involving the left main or proximal LAD segment are relatively rarely qualified for surgical treatment.

\section{Conflict of interest}

The authors declare no conflict of interest.

\section{Corresponding author:}

Piotr M. Wańczura MD, PhD, Department of Cardiology, The Ministry of Internal Affairs and Administration, Hospital, Rzeszow, Poland, e-mail: p.wanczura@poczta.fm

Received: 29.03.2021, accepted: 5.05.2021. 

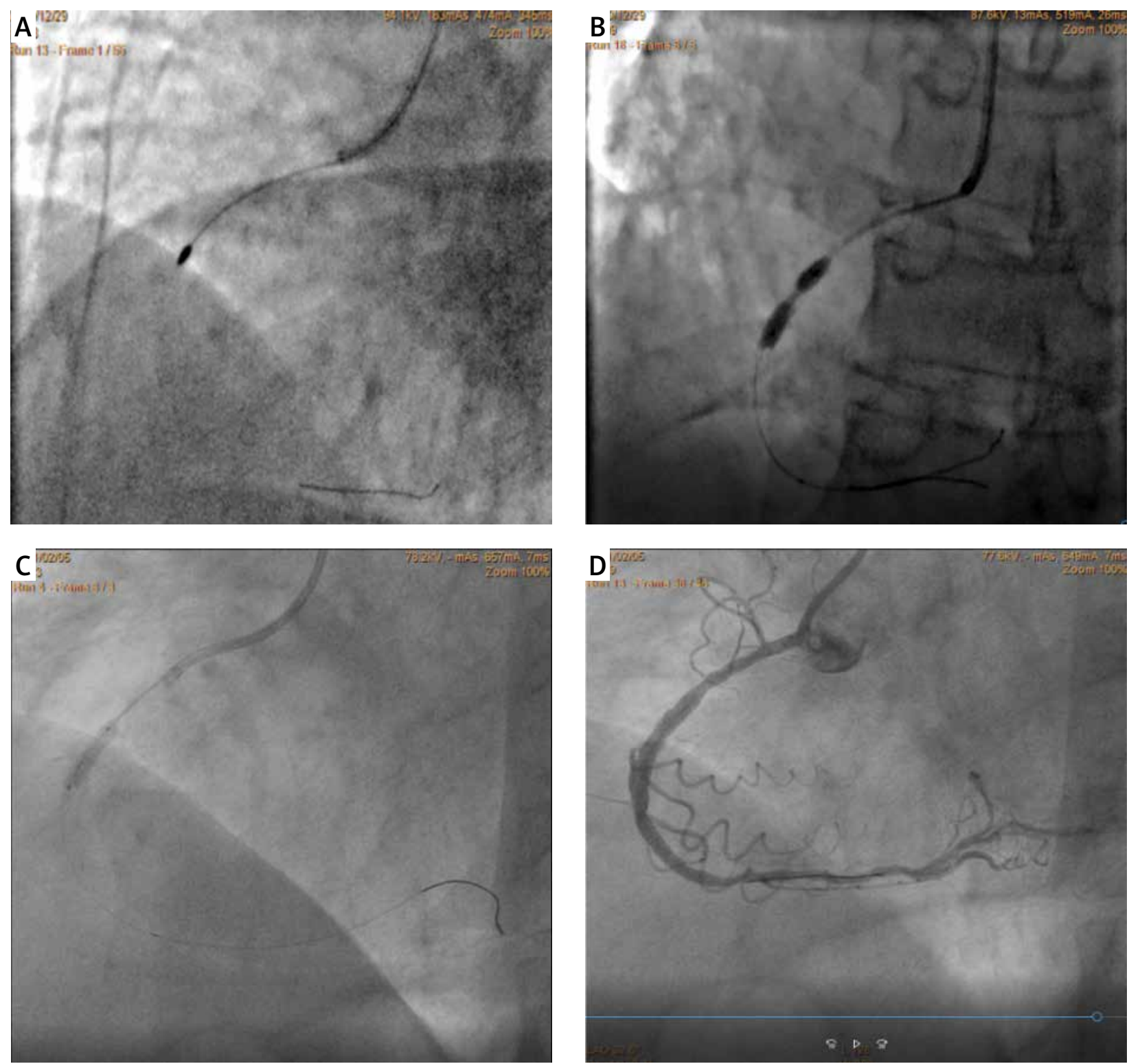

Figure 1. A - Rotational atherectomy with $1.75 \mathrm{~mm}$ burr, B - unexpanded $3.5 \times 20 \mathrm{~mm}$ NC baloon, C - full expanded $3.5 \times 12 \mathrm{~mm} \mathrm{IVL}$ balloon, D - final result after DES implantation

\section{References}

1. Kassimis G, Raina T, Kontogiannis N, et al. How should we treat heavily calcified coronary artery disease in contemporary practice? From atherectomy to intravascular lithotripsy. Cardiovascular Revasc Med 2019; 20: 1172-83.

2. Dini CS, Tomberli B, Mattesini A, et al. Intravascular lithotipsy for salcific coronary and peripheral artery stenoses. Eurointervention 2019; 15: 714-21. 